\title{
DETERMINATION OF THE TURBULENT TEMPERATURE-HUMIDITY CORRELATION FROM SCINTILLOMETRIC MEASUREMENTS
}

\author{
ANDREAS LÜDI ${ }^{1, *}$, FRANK BEYRICH $^{2}$ and CHRISTIAN MÄTZLER ${ }^{1}$ \\ ${ }^{1}$ Institute of Applied Physics, University of Bern, 3012Bern, Switzerland; \\ ${ }^{2}$ Meteorological Observatory Lindenberg, German Meteorological Service (DWD), 15864 \\ Lindenberg, Germany
}

(Received in final form 9 December 2004)

\begin{abstract}
We report on the investigation and successful application of the bichromatic correlation of optical and microwave signals for determining the area-averaged correlation of temperature-humidity fluctuations. The additional technical effort is marginal compared to the common 'two-wavelength method', which has (in contrast) the restriction that only two of the three relevant meteorological structure parameters can be deduced. Therefore, in the past, it was often assumed that the turbulent humidity and temperature fluctuations are perfectly positively or negatively correlated. However, as shown in this study, over non-homogeneous terrain when the flow conditions are not ideal, this assumption is questionable. The measurements were analysed statistically, and were compared to in situ measurements of the Bowen ratio $B o$ and the correlation of temperature-humidity fluctuations using eddycovariance techniques. The latter is in good agreement to that derived by scintillometry. We found that the correlation is not \pm 1 but as low as -0.6 for $B o$ smaller than -2 , and up to 0.8 for $B o$ larger than 1 .
\end{abstract}

Keywords: Area-averaged fluxes, Bichromatic correlation, Correlation of temperaturehumidity fluctuations, Electromagnetic waves, Heterogeneous land surface, Microwave and optical scintillometer.

\section{Introduction}

Many applications in meteorology and the atmospheric sciences demand continuous measurements of turbulent surface heat fluxes that are representative of extended areas. Over heterogeneous terrain, local point measurements, using profile or eddy-covariance techniques, are not suitable, and it has been found that scintillometry appears to be a reliable method for measuring spatially averaged fluxes (e.g. Beyrich et al., 2002; Meijninger et al., 2002a, 2002b, and references therein).

In order to measure sensible and latent heat fluxes using scintillometry, several investigations have been carried out to date by applying the so-called

\footnotetext{
* E-mail: andreas.luedi@armasuisse.ch
} 
two-wavelength method (e.g. Kohsiek and Herben, 1983; Hill et al., 1988; Andreas, 1989; Hill, 1997; Green et al., 2000, 2001; Meijninger et al., 2002a). With this method the path-averaged refractive index structure parameter $C_{n}^{2}$ is measured at two wavelengths $\lambda_{i}$ simultaneously. The parameter $C_{n}^{2}$ can be expressed as

$$
C_{n_{i}}^{2}=A_{i}^{2} C_{T}^{2}+2 A_{i} B_{i} C_{T q}+B_{i}^{2} C_{q}^{2},
$$

with the three unknowns, the temperature structure parameter $C_{T}^{2}$, the (specific) humidity structure parameter $C_{q}^{2}$ and the temperature-humidity structure parameter $C_{T q}$. The constants $A_{i}=A\left(\lambda_{i}, P, T, q\right)$ and $B_{i}=B\left(\lambda_{i}, P, T\right.$, q) depend on the mean temperature $(T)$, the air pressure $(P)$, the specific humidity $(q)$ and the electromagnetic wavelength $\left(\lambda_{i}\right)$. With measurements of $C_{n}^{2}$ at only two wavelengths, it is impossible to find all three meteorological structure parameters, which is a serious disadvantage of the two-wavelength method. Thus it can only be applied by assuming that the three parameters are not independent, and it is often assumed that $C_{T_{q}}= \pm\left(C_{T}^{2} C_{q}^{2}\right)^{1 / 2}$, implying that the correlation coefficient

$$
r_{T q}=\frac{C_{T q}}{\sqrt{C_{T}^{2} C_{q}^{2}}}
$$

between temperature and humidity fluctuations is assumed to be \pm 1 (e.g. Kohsiek and Herben, 1983; Hill et al., 1988; Andreas, 1989; Hill, 1997; Green et al., 2000, 2001; Meijninger et al., 2002a). In fact, this assumption is theoretically demanded for a flow that strictly obeys the Monin-Obukhov similarity theory (MOST) (Hill, 1989). For non-ideal flow conditions, the assumption of $C_{T_{q}}= \pm \sqrt{C_{T}^{2} C_{q}^{2}}$ is not always justifiable, and, especially over non-homogeneous terrain, the strict validity of MOST and hence the perfect temperature-humidity correlation is rather questionable (Andreas, 1987; Andreas et al., 1998; De Bruin et al., 1993). Therefore it would be a considerable advantage to know also the parameter $C_{T q}$ independently. Therewith more accurate determinations of $C_{T}^{2}$ and $C_{q}^{2}$ should be feasible, leading to better heat flux estimates. Furthermore, it is thought that scalar-scalar correlations are especially sensitive to violations of MOST (Hill, 1989; Andreas et al., 1998). Thus, measurements of $C_{T q}$, together with $C_{T}^{2}$ and $C_{q}^{2}$, would also be well suited to answering fundamental questions about the MOST validity and applicability.

In order to determine all three meteorological structure parameters in (1) three independent $C_{n}^{2}$ measurements should be available; this demand could be met by the 'three-wavelength method' (Phelps and Pond, 1971; Hill et al., 1988; Andreas, 1990; Hill, 1997), Unfortunately, it is not easy to find three independent wavelengths in available electromagnetic spectral regions. In 
addition, Andreas (1990) showed theoretically that the accuracy of $C_{T q}$ by this method is poor, and it would be a rather costly experimental exercise, since at least three scintillometers are needed. These may be reasons why the three-wavelength method never has been experimentally applied.

As suggested by Lüdi (2002), another possibility of measuring pathaveraged values of $C_{T q}$ is by cross-correlating two electromagnetic signals at different wavelengths that pass through the same volume of air. Thereby the effects of refractive dispersion on the bichromatic correlation have to be taken into account. Because only the powers (or the intensities) of the two signals (and not the electromagnetic fields) must be correlated, the additional technical effort is marginal compared to the common two-wavelength method.

The cross-correlation between two signals at different wavelengths has already been discussed (Hill, 1988; Hill and Lataitis, 1989) in order to determine the inner scale of turbulence. However, to our knowledge, the determination of $C_{T q}$ through this technique has not been previously analysed and tested.

In this paper, we present measurements collected from 15 April 2003 to 29 May 2003 in Lindenberg (Germany), carried out in clear air conditions (with the propagation path free of hydrometeors) with two scintillometers at wavelengths of $940 \mathrm{~nm}$ and $3.2 \mathrm{~mm}$. With the correlation of the two signals, we infer therefrom three quantities, the two monochromatic variances and the bichromatic covariance, by which $C_{T}^{2}, C_{q}^{2}$ and $C_{T q}$ can be extracted. The detailed description of our experimental set-up is outlined in Section 2, and the theoretical derivation of the measurable quantities is given in Section 3. A sensitivity analysis of the measurable quantities is given in Section 4. The measured correlation of path-averaged temperature-humidity fluctuations is discussed in Section 5, followed by conclusions in Section 6.

As far as we know, the current investigation presents the first path-averaged measurements of turbulent temperature-humidity correlations.

\section{Description of the Experiment}

The study region is a heterogeneous landscape around the Meteorological Observatory Lindenberg of the Deutscher Wetterdienst (DWD) $65 \mathrm{~km}$ south-east of Berlin, Germany. The land use in the area is composed of forest $(42 \%)$, agricultural fields $(41 \%)$, lakes $(6.5 \%)$, meadows $(5 \%)$ and villages $(3.5 \%)$, and is quite typical of large parts of northern central Europe south of the Baltic Sea. A more detailed description of the landscape (including maps) is given in Beyrich et al. (2002).

As mentioned in Section 1, two scintillometers, one in the optical to nearinfrared region (wavelength $\lambda=940 \mathrm{~nm}$ ) and a second in the millimeter 
region $(\lambda=3.2 \mathrm{~mm})$, are operated over a path length of $4.7 \mathrm{~km}$ in a northsouth orientation. The microwave and optical scintillometers have antenna diameters of 0.4 and $0.15 \mathrm{~m}$, respectively, which are identical for transmitters and receivers. Both scintillometers have diverging beams with full power beam widths of approximately 1 degree, and have been installed on two permanent towers. Many technical details about the optical instruments can be found in Moene et al. (2005). The receivers (north end of path) are mounted at the top of a $30-\mathrm{m}$ tower and the transmitters are installed at a height of $50 \mathrm{~m}$ on the $99-\mathrm{m}$ meteorological tower near Falkenberg. The effective height of the propagation path is $45 \mathrm{~m}$ above ground (Beyrich et al., 2002).

As mentioned above, both electromagnetic beams are required to cross the same air volume and therefore the two beams should be as close to each other as possible. If both beams are focused by the same antenna dishes, we obtain the best possible configuration. However, this set-up usually cannot be realised, and an acceptable and realistic set-up is shown in Figure 1. The distance between the optical axis of both transmitters should be as small as possible, and the same is true for the receivers; additionally, the beams should intersect in the middle of the path. In our set-up this latter configuration was realised only approximately, as the mounting possibilities on the 99-m tower were critical. The relative positions of the transmitters and receivers with respect to each other are $\boldsymbol{d}_{\boldsymbol{T}}=(y, z)=(0.25,-1) \mathrm{m}$ and $\boldsymbol{d}_{\boldsymbol{R}}=(y, z)=(0,0.5) \mathrm{m}$ (cf. Figure 1). As will be shown in the next section, the performance of this set-up is not much worse than the optimal configuration.

For the analysis presented here, measurement periods of 10 min were used. This choice is a compromise between stationarity and statistical significance (Caccia et al., 1987); if the period is too long, the assumption of atmospheric stationarity may be violated, and if the period is too short the statistical weight of the estimations is very poor.

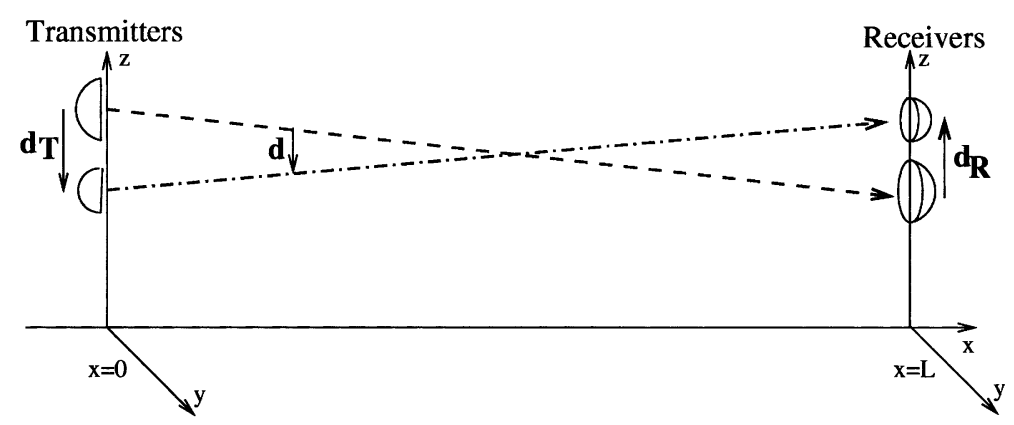

Figure 1. Schematic view of the measurement configuration. The microwave and optical beams are shown as dashed-dotted lines, respectively. The relative positions of the transmitters and receivers are $\boldsymbol{d}_{\boldsymbol{T}}=(y, z)=(0.25,-1) \mathrm{m}$ and $\boldsymbol{d}_{\boldsymbol{R}}=(y, z)=(0,0.5) \mathrm{m}$. 
The data shown herein were obtained during clear air conditions. Thus, any data obtained during precipitation or during fog (leading to a low optical signal) were rejected. In addition, according to the saturation criteria of Ochs and Hill (1982) and Wang et al. (1978), data that may be contaminated by saturation of scintillation were eliminated. In this study also, measured vertical gradients of specific humidity and temperature are used (cf. Section 5). As several in situ meteorological stations are available we also rejected measurements when the gradients derived by different stations were not equal to within a factor of 2 .

Before computing the variances and the covariance of the measured signals, we bandpass filtered them. The optical signal was split into two parts, which were differently filtered; one part of the optical signal was used to determine the signal variance and the other part was used to determine the cross-covariance between optical and microwave signals. As the latter part of the optical signal was filtered the same as the microwave signal it is not discussed explicitly. The upper frequency cut-offs of the signals are determined by the receiving hardware: the received microwave signal was low-pass filtered at $f=20 \mathrm{~Hz}$ and sampled at $f=40 \mathrm{~Hz}$ (Nyquist theorem). The respective frequencies for the optical signal are higher by a factor of 20 . According to the theoretical model (Section 3 and Figure 3), scintillations at microwave wavelengths dominate for $0.04 \lesssim f / v \lesssim 10 \mathrm{~m}^{-1}$ and scintillations at optical wavelengths for $0.1 \lesssim f / v \lesssim 100 \mathrm{~m}^{-1}$, with $f$ the temporal frequency and $v$ the wind velocity component transverse to the propagation path. With $v$ equal to several $\mathrm{m} \mathrm{s}^{-1}$, signal fluctuations due to turbulence are expected at $\lambda_{1}=940 \mathrm{~nm}$ only in the range of $0.2<f<400 \mathrm{~Hz}$ and at $\lambda_{2}=3.2 \mathrm{~mm}$ for 0.06 $<f<20 \mathrm{~Hz}$. These ranges hence reveal the bandpass filtering of the signals. The suppression of low-frequency fluctuations eliminates gain drifts of the instruments and changes of the atmospheric opacity. Furthermore, as shown in Appendix B, absorption-induced fluctuations are also suppressed. The filtered signals are used thereafter to compute the 10-min averaged variances and the covariance. These values will be compared with the theoretical expressions, to be derived in the following section.

\section{Theoretical Background}

\subsection{The COVARIANCE FUnCtion OF POWER FluCtUATIONS}

The signals are collected by receivers with extended apertures, where the collected power $P$ of a receiver is the integrated intensity $I$ over the aperture area of the incident electromagnetic wave. Thus the covariance function $B_{P}$ of the normalised power $P$ detected by two receivers, with collecting circular apertures $\sum_{R 1}$ and $\sum_{R 2}$ of radius $R_{R 1}$ and $R_{R 2}$, is given by 


$$
\begin{aligned}
B_{P}\left(k_{1}, k_{2}\right) & \doteq \frac{\left\langle P_{1}^{\prime}\left(k_{1}\right) P_{2}^{\prime}\left(k_{2}\right)\right\rangle}{\left\langle P_{1}\left(k_{1}\right)\right\rangle\left\langle P_{2}\left(k_{2}\right)\right\rangle} \\
& =\left(\frac{1}{\pi^{2} R_{R 1}^{2} R_{R 2}^{2}}\right) \int_{\Sigma_{R 1}} \int_{\Sigma_{R 2}} \frac{\left\langle I_{1}^{\prime}\left(k_{1}, \boldsymbol{r}_{1}\right) I_{2}^{\prime}\left(k_{2}, \boldsymbol{r}_{2}\right)\right\rangle}{\left\langle I_{1}\left(k_{1}\right)\right\rangle\left\langle I_{2}\left(k_{2}\right)\right\rangle} d^{2} \boldsymbol{r}_{1} d^{2} \boldsymbol{r}_{2}
\end{aligned}
$$

with the electromagnetic wavenumbers $k_{1}$ and $k_{2}$ of the measured powers $P_{1}$ and $P_{2}$, respectively and $d^{2} \boldsymbol{r}$ is the area element of integration. The power and intensity fluctuations are defined as $P^{\prime}=P-\langle P\rangle$ and $I^{\prime}=I-\langle I\rangle$, with $\langle\ldots\rangle$ representing the ensemble average (here given by the average over the sample period). The vectors $\boldsymbol{r}_{1}$ and $\boldsymbol{r}_{2}$ describe the receiving apertures in the plane $x=L$, with $L$ the length of the propagation path. The intensity $I$, which, in our case, originates from an extended source, can be treated in the same way as the receivers (reciprocity theorem), i.e. as a sum of intensities $J$ from point sources at positions $\rho$ (e.g. Ochs and Wang, 1978). The normalised intensity covariance function (integrand in Equation (3)) is then given by

$$
\frac{\left\langle I_{1}^{\prime}\left(k_{1}, \boldsymbol{r}_{1}\right) I_{2}^{\prime}\left(k_{2}, \boldsymbol{r}_{2}\right)\right\rangle}{\left\langle I_{1}\left(k_{1}\right)\right\rangle\left\langle I_{2}\left(k_{2}\right)\right\rangle}=\frac{\left\langle\int_{\Sigma_{T 1}} d^{2} \boldsymbol{\rho}_{1} J_{1}^{\prime}\left(k_{1}, \boldsymbol{\rho}_{1}, \boldsymbol{r}_{1}\right) \int_{\Sigma_{T 2}} d^{2} \boldsymbol{\rho}_{2} J_{2}^{\prime}\left(k_{2}, \boldsymbol{\rho}_{2}, \boldsymbol{r}_{2}\right)\right\rangle}{\left\langle\int_{\Sigma_{T 1}} d^{2} \boldsymbol{\rho}_{1} J_{1}\left(k_{1}, \boldsymbol{\rho}_{1}\right)\right\rangle\left\langle\int_{\Sigma_{T 2}} d^{2} \boldsymbol{\rho}_{2} J_{2}\left(k_{2}, \boldsymbol{\rho}_{2}\right)\right\rangle},
$$

In our application, the sources $T 1$ and $T 2$ are circular transmitters with radius $R_{T 1}$ and $R_{T 2}$, respectively. A theoretical examination with the wellknown weak fluctuation theory is appropriate because in the $\mathrm{mm}$ range, scintillation is weak. Also at the optical wavelength scintillation is mostly weak, as both the receiver and the transmitter have large apertures. Nevertheless, as noted in Wang et al. (1978), as well as in Ochs and Hill (1982), strong scintillation with saturation effects can occur. Therefore, data from the optical scintillometer that do not satisfy the saturation criteria of Ochs and Hill (1982) and Wang et al. (1978) (for our set-up: $C_{n}^{2} \leqslant 1.4 \times 10^{-14} \mathrm{~m}^{-2 / 3}$ ) have to be rejected as the applied theory is not appropriate.

Interchanging the ensemble averages with the integrals (due to the linearity of integration and ensemble average operators), using $J \propto e^{2 \chi}$ (with $\chi$ the log-amplitude, cf. Tatarskii, 1971) in (4) and presuming $4\left\langle\chi_{1}\left(k_{1}, \boldsymbol{\rho}_{1}, \boldsymbol{r}_{1}\right)\right.$. $\left.\chi^{2}\left(k_{2}, \boldsymbol{\rho}_{2}, \boldsymbol{r}_{2}\right)\right\rangle \ll 1$ (due to the weak fluctuation approximation) we obtain:

$$
\begin{aligned}
B_{p}\left(k_{1}, k_{2}\right) & =\left(\frac{4}{\pi^{4} R_{R 1}^{2} R_{R 2}^{2} R_{T 1}^{2} R_{T 2}^{2}}\right) \\
& \times \int_{\Sigma_{R 1}} \int_{\Sigma_{R 2}} \int_{\Sigma_{T 1}} \int_{\Sigma_{T 2}} d^{2} \boldsymbol{r}_{1} d^{2} \boldsymbol{r}_{2} d^{2} \boldsymbol{\rho}_{1} d^{2} \boldsymbol{\rho}_{2} B_{\chi}\left(k_{1}, k_{2}\right), \\
B_{\chi}\left(k_{1}, k_{2}\right) & =\left\langle\chi_{1}\left(k_{1}, \boldsymbol{\rho}_{1}, \boldsymbol{r}_{1}\right) \chi_{2}\left(k_{2}, \boldsymbol{\rho}_{2}, \boldsymbol{r}_{2}\right)\right\rangle .
\end{aligned}
$$


The log-amplitude correlation function $B_{\chi}\left(k_{1}, k_{2}\right)$ of two electromagnetic waves, neglecting dispersion effects, propagating through a turbulent medium, is well-known (e.g. Ishimaru, 1978, p. 401). From the latter, the extension of taking into account the effects of dispersion is straightforward. It is found for spherical wave propagation that (Hill and Lataitis, 1989)

$$
\begin{aligned}
& B_{\chi}\left(k_{1}, k_{2}\right)=2 \pi k_{1} k_{2} \int_{0}^{L} d x \iint_{-\infty}^{\infty} d^{2} \boldsymbol{\kappa} H \cdot e^{j \boldsymbol{} \mathbf{D}} \Phi_{n_{1} n_{2}}(\boldsymbol{\kappa}), \\
& H=\sin \left(\frac{\gamma(L-x) \kappa^{2}}{2 k_{1}}\right) \sin \left(\frac{\gamma(L-x) \kappa^{2}}{2 k_{2}}\right),
\end{aligned}
$$

where $\boldsymbol{D}=\boldsymbol{\rho}^{\prime \prime}+\gamma\left(\boldsymbol{r}^{\prime \prime}-\boldsymbol{\rho}^{\prime \prime}\right), \boldsymbol{\rho}^{\prime \prime}=\boldsymbol{\rho}_{1}-\boldsymbol{\rho}_{2}, \boldsymbol{r}^{\prime \prime}=\boldsymbol{r}_{1}-\boldsymbol{r}_{2}$. Furthermore we have set $\gamma=x / L$ and $\kappa=\left(\kappa_{y}, \kappa_{z}\right)$ is the two-dimensional spatial wavenumber. The refractive index cospectrum $\Phi_{n_{1} n_{2}}(\kappa)$ is assumed to be locally homogeneous and isotropic. Note, that absorption-induced fluctuations are not taken into account in (7) because they are negligible as shown in Appendix B. Due to the fact that the two transmitters $T 1$ and $T 2$ are centred at different locations in the $(x=0)$-plane it is convenient to decompose $\boldsymbol{\rho}_{i}$ for each transmitter by $\overline{\boldsymbol{\rho}}_{i}+\boldsymbol{\rho}_{i}^{\prime}$, with $\overline{\boldsymbol{\rho}}_{i}$ the centre position of the transmitter $T i$. Then the integrals over the transmitter areas $\sum_{T 1}$ and $\sum_{T 2}$ can be solved by

$$
\int_{\Sigma_{T i}} d^{2} \boldsymbol{\rho}_{i}^{\prime} e^{ \pm j \boldsymbol{\kappa} \rho_{i}^{\prime}(1-\gamma)}=2 \pi R_{T i}^{2} \frac{J_{1}\left(\boldsymbol{\kappa}(1-\gamma) R_{T i}\right)}{\boldsymbol{\kappa}(1-\gamma) R_{T i}}, \quad i=1,2,
$$

where $J_{1}$ is the Bessel function of the first kind of order one. For the same reason we also decompose $\boldsymbol{r}_{\boldsymbol{i}}=\overline{\boldsymbol{r}}_{i}+\boldsymbol{r}_{i}^{\prime}$, allowing to solve the integrals over the receiving apertures $\Sigma_{R 1}$ and $\Sigma_{R 2}$. The assumption of isotropy allows us to integrate (7) over direction, i.e.

$$
\begin{aligned}
& \iint_{-\infty}^{\infty} d^{2} \boldsymbol{\kappa} e^{j \boldsymbol{k} \boldsymbol{d}}=2 \pi \int_{0}^{\infty} d \kappa \kappa J_{0}(\kappa|\boldsymbol{d}|), \\
& d=(1-\gamma) \boldsymbol{d}_{\boldsymbol{T}}+\gamma \boldsymbol{d}_{\boldsymbol{R}},
\end{aligned}
$$

where $J_{0}$ is the Bessel function of the first kind of order zero, $\boldsymbol{d}_{T}=\overline{\boldsymbol{\rho}}_{1}-\overline{\boldsymbol{\rho}}_{2}$ is the distance between the transmitters, and $\boldsymbol{d}_{\boldsymbol{R}}=\overline{\boldsymbol{r}}_{1}-\overline{\boldsymbol{r}}_{2}$ the separation of the receivers (cf. Figure 1).

The refractive index cospectrum $\Phi_{n_{1} n_{2}}(\kappa)$ is related to the power spectra of temperature and specific humidity fluctuations, $\Phi_{T}(\kappa)$ and $\Phi_{q}(\kappa)$, and the temperature-humidity cospectrum $\Phi_{T q}(\kappa)$, by (Hill et al., 1980)

$$
\Phi_{n_{1} n_{2}}(\kappa)=A_{1} A_{2} \Phi_{T}(\kappa)+\left(A_{1} B_{2}+A_{2} B_{1}\right) \Phi_{T q}(\kappa)+B_{1} B_{2} \Phi_{q}(\kappa) .
$$


The constants $A_{i}$ and $B_{i}$ are the same as in Equation (1). In the inertial subrange of turbulence it is generally accepted that all of the spectra in (10) are proportional to $0.033 \kappa^{-11 / 3}$ (three-dimensional Kolmogorov spectrum) with the respective structure and cross-structure parameters as the coefficients. Thus

$$
C_{n_{1} n_{2}}=A_{1} A_{2} C_{T}^{2}+\left(A_{1} B_{2}+A_{2} B_{1}\right) C_{T q}+B_{1} B_{2} C_{q}^{2} .
$$

For $\lambda_{1}=\lambda_{2}$, (11) coincides with (1). Note that the dissipation subrange of turbulence is suppressed due to the use of extended receivers and transmitters. Also the large-scale range (production or input range) of turbulence is not observable due to the high-pass filtering effect of the expression $H$ (cf. Equation (8)). Thus, effects of inhomogeneity scales larger than the Fresnel size $\sqrt{\lambda L}$ of the mm-wave scintillometer (i.e. larger than approximately $4 \mathrm{~m}$ ) are suppressed. As the inertial subrange of turbulence extends to considerably larger scales than only $4 \mathrm{~m}$ (particularly at $45 \mathrm{~m}$ above ground) the adoption of the Kolmogorov spectrum $\left(\propto \kappa^{-11 / 3}\right)$ is reasonable and justified.

With the refractive cospectrum $\Phi_{n_{1} n_{2}}(\kappa)=0.033 C_{n_{1} n_{2}} \kappa^{-11 / 3}$ the expression for $B_{P}$ finally becomes:

$$
B_{P}(\boldsymbol{d})=8.448 \pi^{2} k_{1} k_{2} \int_{0}^{L} d x C_{n_{1} n_{2}} W(\boldsymbol{d}, x),
$$

where the weighting function $W$ is given by

$$
\begin{aligned}
& W(\boldsymbol{d}, x)=\int_{0}^{\infty} d \kappa \kappa^{-8 / 3} H \cdot F \cdot J_{0}(\kappa|\boldsymbol{d}|), \\
& F=\frac{J_{1}\left(\kappa(1-\gamma) R_{T_{1}}\right) J_{1}\left(\kappa(1-\gamma) R_{T_{2}}\right) J_{1}\left(\kappa \gamma R_{R 1}\right) J_{1}\left(\kappa \gamma R_{R 2}\right)}{\kappa^{4} R_{R 1} R_{R 2} R_{T 1} R_{T 2}(1-\gamma)^{2} \gamma^{2}}, \\
& \gamma=x / L .
\end{aligned}
$$

Equation (12) describes the covariance function of the power fluctuations for two extended transmitters with radius $R_{T 1}$ and $R_{T 2}$ separated by $\boldsymbol{d}_{T}$, each observed by individual receivers, separated by $\boldsymbol{d}_{\boldsymbol{R}}$, collecting the signals through apertures of radius $R_{R 1}$ and $R_{R 2}$, respectively. The distances $\boldsymbol{d}_{\boldsymbol{T}}$ and $\boldsymbol{d}_{\boldsymbol{R}}$ are related to $\boldsymbol{d}$ by (9). The expression $F$ describes the averaging effects due to the extended transmitter and receiver apertures. Let us mention that the aperture averaging effects are significant only for the optical signal. At microwaves, they are negligible since the Fresnel zone $(\sqrt{\lambda L})$ is much larger (by a factor of more than 10) than the aperture radius. 


\subsection{WeIGHTING FUnCTIONS}

Equation (12) is the basic equation in our analysis to infer meteorological structure parameters from scintillation. With our measuring set-up (cf. Sections 1 and 2) we measure three signals, namely the cross-covariance of the detected power of the two signals at different wavelengths and the two monochromatic variances $\left(\lambda=\lambda_{2}\right.$ for $\boldsymbol{d}=0$ in Equation (12)), yielding path averaged $C_{n_{1} n_{2}}, C_{n_{1}}^{2}$ and $C_{n_{2}}^{2}$. The relative weighting of these latter parameters along the path is described by the weighting function $W(\boldsymbol{d}, x)$ which depends on $\boldsymbol{d}$ (Equation (9)). In Figure 2 the function $W(0, x)$, normalised to $W(0$, $x=L / 2$ ), for $C_{n_{1}}^{2}$ and $C_{n_{2}}^{2}$ are shown as dashed-dotted and solid lines, respectively. The parameters for computing the curves are as in our experiment; $L=4.7 \mathrm{~km}, \lambda_{1}=940 \mathrm{~nm}, \lambda_{2}=3.2 \mathrm{~mm}, R_{R 1}=R_{T 1}=0.075 \mathrm{~m}$ and $R_{R 2}$ $=R_{T 2}=0.2 \mathrm{~m}$. The significant reduction of the weighting function for $C_{n_{1}}^{2}$ close to the receiver and transmitter is due to the fact that the ratio of aperture radius and Fresnel zone $(\sqrt{\lambda L})$ is larger than 1 .

The normalised weighting function for $C_{n_{1} n_{2}}$ should also be similar to the former ones, in particular it should be also maximal in the middle of the path. In addition, in order to have a strong signal, the integral of $W(\boldsymbol{d}, x)$ should be as large as possible. These demands are best met by minimising the distance $\boldsymbol{d}$. This means that $\boldsymbol{d}_{\boldsymbol{T}}$ and $\boldsymbol{d}_{\boldsymbol{R}}$ should be as small as possible, and the two beams should cross each other in the middle of the path (where $\boldsymbol{d}=0$, cf. Figure 1). Computed weighting functions of $C_{n_{1} n_{2}}$ are shown as dotted and

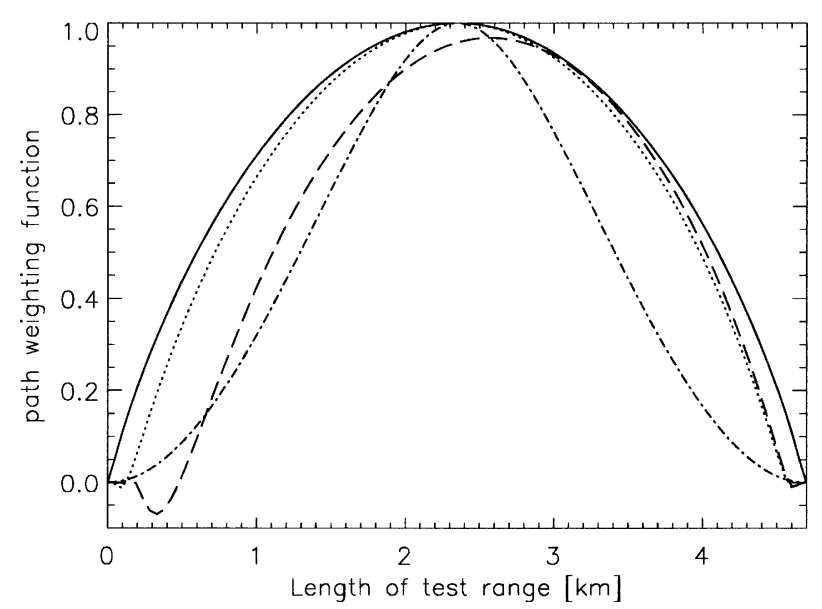

Figure 2. Normalised weighting functions $W(\boldsymbol{d}, x)$ for $C_{n 1 n 2}$ for different separations of the receivers and transmitters are shown for relative positions of $\boldsymbol{d}_{T}=(0.25,-1) \mathrm{m}$ and $d_{R}=(0$, $0.5) \mathrm{m}$ (dashed, actual set-up) and $\boldsymbol{d}_{\boldsymbol{T}}=-\boldsymbol{d}_{\boldsymbol{R}}=(0,0.5) \mathrm{m}$ (dotted). Normalised weighting functions $W(0, x)$ for $C_{n_{1}}^{2}(\lambda=940 \mathrm{~nm})$ and $C_{n_{2}}^{2}(\lambda=3.2 \mathrm{~mm})$ are shown as dashed-dotted and solid lines, respectively. 
dashed lines in Figure 2, The dotted curve results from $\boldsymbol{d}_{T}=(0,0.5)$ and $\boldsymbol{d}_{\boldsymbol{R}}=(0,-0.5)$ (metres) and shows the best possible realisation that could be achieved with our receivers and transmitters. The dashed curve results from $\boldsymbol{d}_{\boldsymbol{T}}=(0.25,-1.0)$ and $\boldsymbol{d}_{\boldsymbol{R}}=(0,0.5)$, representing the actual set-up. The weighting functions of $C_{n_{1} n_{2}}$ in Figure 2 are again normalised to $W(\boldsymbol{d}=0$, $x=L / 2$ ), i.e. normalised to the maximum of the dotted curve. The dotted curve deviates only slightly from the solid curve, thus $C_{n_{1} n_{2}}$ would almost be equally weighted with this configuration as $C_{n_{2}}^{2}$. As mentioned above, in our experiment it was not possible to mount the transmitters perfectly, and the two beams do not intersect in the middle of the path. Therefore, the maximum is slightly reduced and shifted toward the receivers. Nevertheless the maximum of the weighting function for our realisation (dashed curve) is still close to the middle of the path and all three parameters $C_{n_{1}}^{2}, C_{n_{2}}^{2}$ and $C_{n_{1} n_{2}}$ are averaged basically over the same air volume.

Replacing $C_{n_{1}}^{2}, C_{n_{2}}^{2}$ and $C_{n_{1} n_{2}}$ by representative mean values along the path $x$, Equation (12) can also be written as

$$
\begin{aligned}
& B_{P}=\int_{0}^{\infty} d \kappa G(\kappa) C_{n_{1} n_{2}}, \\
& G(\kappa)=8.448 \pi^{2} k_{1} k_{2} \int_{0}^{L} d x \kappa^{-8 / 3} H \cdot F \cdot J_{0}(\kappa|\boldsymbol{d}|) .
\end{aligned}
$$

The spectral filter functions $G(\kappa)$ for the optical, the microwave and the covariance signals are given as dashed, solid and dotted lines, respectively in Figure 3, showing the relative contribution of inhomogeneity scales to the

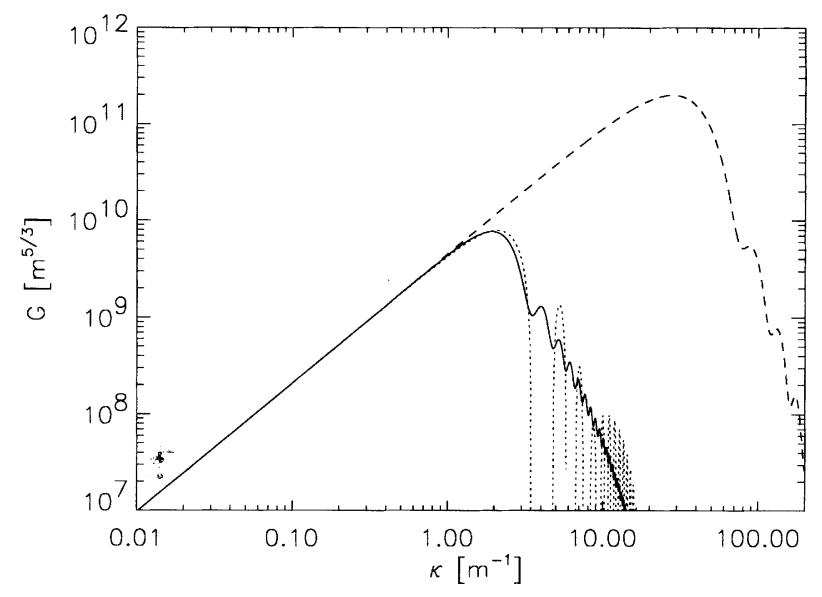

Figure 3. Spectral filter functions for $C_{n_{1}}^{2}$ (dashed), $C_{n_{2}}^{2}$ (solid) and $C_{n_{1} n_{2}}$ (dotted). The refractivities $n_{1}$ and $n_{2}$ stand for $\lambda_{1}=940 \mathrm{~nm}$ and $\lambda_{2}=3.2 \mathrm{~mm}$, respectively. 
measured signals. At the optical wavelength $G(\kappa)$ extends to higher spatial frequencies $\kappa$; therefore the temporal frequency bandpass (before the detection of the signal) was chosen to be higher than at the other two signals (cf. Section 2). The filters $G(\kappa)$ for the microwave and covariance signals are almost equal. Therefore, we filtered the corresponding measured signals equally (cf. Section 2).

\subsection{Meteorological structure parameters from $C_{n_{1}}^{2}, C_{n_{2}}^{2}$ ANd $C_{n_{1} n_{2}}$}

From the measured refractive structure parameters $C_{n_{1}}^{2}, C_{n_{2}}^{2}$ and $C_{n_{1} n_{2}}$ the meteorological structure parameters, $C_{T}^{2}, C_{q}^{2}$ and $C_{T q}$ can be inferred. From (1) and (11) we obtain

$$
C_{p}=M C_{s}
$$

with

$$
\boldsymbol{M}=\left(\begin{array}{ccc}
A_{1}^{2} & 2 A_{1} B_{1} & B_{1}^{2} \\
A_{2}^{2} & 2 A_{2} B_{2} & B_{2}^{2} \\
A_{1} A_{2} & \left(A_{1} B_{2}+A_{2} B_{1}\right) & B_{1} B_{2}
\end{array}\right),
$$

where $C_{\boldsymbol{s}}$ and $C_{\boldsymbol{p}}$ are vectors with components $\left(C_{T}^{2}, C_{T q}, C_{q}^{2}\right)$ and $\left(C_{n_{1}}^{2}, C_{n_{2}}^{2}\right.$, $C_{n_{1} n_{2}}$ ), respectively, Equation (14) can be inverted if

$$
\operatorname{det}[\boldsymbol{M}]=-A_{1}^{3} B_{2}^{3}\left(1-\frac{A_{2} B_{1}}{A_{1} B_{2}}\right)^{3} \neq 0,
$$

requiring $\left(A_{2} B_{1}\right) /\left(A_{1} B_{2}\right) \neq 1$. In the optical region we determined the wavelength-dependent parameters $A_{i}$ and $B_{i}$ with expressions given in Andreas (1988) and in the mm-wavelength region we used the MillimeterWave Propagation Model (MPM93) of Liebe et al. (1993). MPM93, also known as the "Liebe Model", was recently experimentally validated at $94 \mathrm{GHz}$ and good agreement between the modelled data and the observations was found for the clear atmosphere (Martin et al., 2000). For the present wavelengths and typical meteorological conditions $\left(T=20^{\circ} \mathrm{C}\right.$, $P=1000 \mathrm{hPa}$ and $90 \%$ relative humidity) the ratio $\left(A_{2} B_{1}\right) /\left(A_{1} B_{2}\right)=-0.01$. The inverse matrix $\boldsymbol{M}^{-1}$ is given by

$$
\boldsymbol{M}^{-1}=\frac{1}{\left(A_{1} B_{2}-A_{2} B_{1}\right)^{2}}\left(\begin{array}{ccc}
B_{2}^{2} & B_{1}^{2} & -2 B_{1} B_{2} \\
-A_{2} B_{2} & -A_{1} B_{1} & \left(A_{1} B_{2}+A_{2} B_{1}\right) \\
A_{2}^{2} & A_{1}^{2} & -2 A_{1} A_{2}
\end{array}\right) .
$$

Consequently, there exists an unambiguous solution for $C_{T}^{2}, C_{q}^{2}$ and $C_{T q}$, found from measurements of $C_{n_{1}}^{2}, C_{n_{2}}^{2}$ and $C_{n_{1} n_{2}}$ given by

$$
C_{s}=M^{-1} C_{p}
$$




\section{Sensitivity Analysis}

In this section we consider how measurement uncertainties of $C_{n_{1}}^{2}, C_{n_{2}}^{2}$ and $C_{n_{1} n_{2}}$ affect the parameters $C_{T}^{2}, C_{q}^{2}$ and $C_{T q}$. As already mentioned in Section 1, the "three-wavelength method" (Phelps and Pond, 1971; Andreas, 1990; Hill et al., 1988; Hill, 1997) is the only alternative for measuring path-averaged structure parameters, $C_{q}^{2}, C_{T}^{2}$ and $C_{T q}$. In Andreas (1990) a detailed sensitivity analysis of the "three-wavelength method" was carried out. In order to compare the two methods, we performed an analogous sensitivity analysis and use (as far as possible) the same nomenclature.

From (17) it is seen that uncertainties in the measured $C_{p j}$ affect each of the meteorological structure parameters. With the notation already introduced above the absolute uncertainty of $C_{s i}$ can be expressed as

$$
d C_{s i}=\sum_{j=1}^{3} \frac{\partial C_{s i}}{\partial C_{p j}} d C_{p j}
$$

However, it is better to consider the relative uncertainties since the $\boldsymbol{C}_{\boldsymbol{p}}$ values can range over several orders of magnitude. Therefore (18) is modified to (Andreas, 1990)

$$
\begin{aligned}
& \frac{d C_{s i}}{C_{s i}}=\sum_{j=1}^{3} S_{i j} \frac{d C_{p j}}{C_{p j}}, \\
& S_{i j}=\frac{C_{p j}}{C_{s i}} \frac{\partial C_{s i}}{\partial C_{p j}},
\end{aligned}
$$

where $d C_{s i} / C_{s i}$ is the relative uncertainty of the computed meteorological structure parameter, and $d C_{p j} / C_{p j}$ is the relative uncertainty of the measured refractivity structure parameter. In the following the non-dimensional sensitivity coefficients $S_{i j}$ will be referred to as $S_{T j}, S_{q j}$ and $S_{T q j}(j=1, \ldots, 3)$. As shown by Andreas (1990) the sensitivity coefficients can be expressed as functions of the Bowen ratio $B o$ and the temperature-humidity correlation coefficient $r_{T q}$, defined in (2). The Bowen ratio $B o$ is an important parameter to understand the sensitivity $S ; B o$ is defined as the ratio of the sensible to the latent heat fluxes. Since $B o$ has the same sign as $C_{T q}$ and $B o^{2}=\left(c_{p}\langle w T\rangle\right)^{2} /\left(L_{v}\langle w q\rangle\right)^{2}=\left(c_{p}^{2} C_{T}^{2}\right) /\left(L_{v}^{2} C_{q}^{2}\right)$ it follows (Andreas, 1990)

$$
B o=\frac{\operatorname{sgn}\left[C_{T q}\right]}{K} \sqrt{\frac{C_{T}^{2}}{C_{q}^{2}}},
$$

where $w, T$ and $q$ are, respectively, the vertical wind velocity, temperature and specific humidity fluctuations. Further, $K=L_{v} / c_{p}$, where $c_{p}$ is the specific heat of air at constant pressure, and $L_{v}$ is the latent heat of vaporisation or sublimation, 
Strictly speaking, the assumption of $\langle w T\rangle^{2} /\langle w q\rangle^{2}=C_{T}^{2} / C_{q}^{2}$ leading to (21) is only absolutely correct when Monin-Obukhov similarity is valid. With the computed sensitivity functions we know what the relative uncertainties in $C_{T}^{2}$, $C_{q}^{2}$ and $C_{T q}$ will be for given relative uncertainties in the measured refractive index structure parameters. The derivations of the sensitivity functions are straightforward (see also Andreas, 1990), and their explicit expressions are given in Appendix A. They are shown as a function of $B o$ in Figures 4 and 5. Figure 4 shows the curves for $S_{T}$ and $S_{q}$, respectively, with $\left|r_{T q}\right|=1$, where the curves are computed for typical meteorological conditions; $T=20^{\circ} \mathrm{C}, P=1000 \mathrm{hPa}$ and relative humidity of $90 \%$, as also chosen by Andreas (1990). The sensitivity coefficients depend only weakly on the meteorological conditions.

If the $|S|$ values are larger than 1 , the resulting error in the meteorological structure parameter is accordingly larger than the measured refractivity structure parameters. If the $S$ value is close to zero, the respective measure of $C_{p j}$ is not sensitive to the meteorological structure parameter $C_{s i}$ under consideration. Thus, in general, optimum measurements can be made by having two $S$ values close to 0 and the other value close to 1 (or -1 ).

From the sensitivity functions $S_{T j}$ (cf. Figure 4, upper panel) it is seen that $C_{T}^{2}$ is well measurable when $|B o| \gtrsim 0.05$. In this range (except for $-0.2 \lesssim$ $B o \lesssim-0.05) S_{T 1}$ (from the optical scintillometer) is close to 1 whereas the other two $S_{T j}$ are close to 0 . Only for $|B o| \lesssim 0.05$ is $C_{T}^{2}$ is scarcely measurable, This was also found with the "three-wavelength method" (Andreas, 1990).

From the sensitivity functions $S_{q j}$ (cf. Figure 4, lower panel) it is seen that the information is primarily encountered in the microwave signal, as $S_{q 2}$ is approximately 1 , whereas the others are mostly close to 0 . The structure parameter $C_{q}^{2}$ can be well measured since $|B o| \lesssim 3$; otherwise larger errors in $C_{q}^{2}$ have to be taken into account. In this respect the "three-wavelength method" is worse as it is more limited to the range $|B o|<0.5$ (Andreas, 1990).

From the sensitivity functions $S_{T q j}$ (cf. Figure 5, upper panel) it is seen that $C_{T q}$ is much more difficult to measure than the other two meteorological structure parameters. According to $S_{T q 1}$ and $S_{T q 2}$ for $|B o|<1$ even small errors in $C_{n_{1}}^{2}$ and $C_{n_{2}}^{2}$ result in large errors in $C_{T q}$. Measurement errors in $C_{n_{1} n_{2}}$ are not so critical because $\left|S_{T q 3}\right|$ is small. However, our method can be well used in the two windows around $1<|B o|<10$ with $\left|r_{T q}\right|=1$. The lower panel of Figure 5 shows that these two ranges become narrower when $\left|r_{T q}\right|$ is smaller. We also tested other wavelength combinations, in particular the combination with an infrared signal from the window of $\lambda=7.8-19 \mu \mathrm{m}$. When we pair a visible-to-near infrared signal with the latter, the resulting measurability of $C_{T q}$ is completely useless. A little better, but still more-or-less useless, and much worse than our combination, is the combination of a millimeter signal with an infrared signal from the above mentioned window. This shows that even though our sensitivity for measuring $C_{T q}$ is not exhilarating, it cannot simply be improved by choosing other wavelength combinations. 

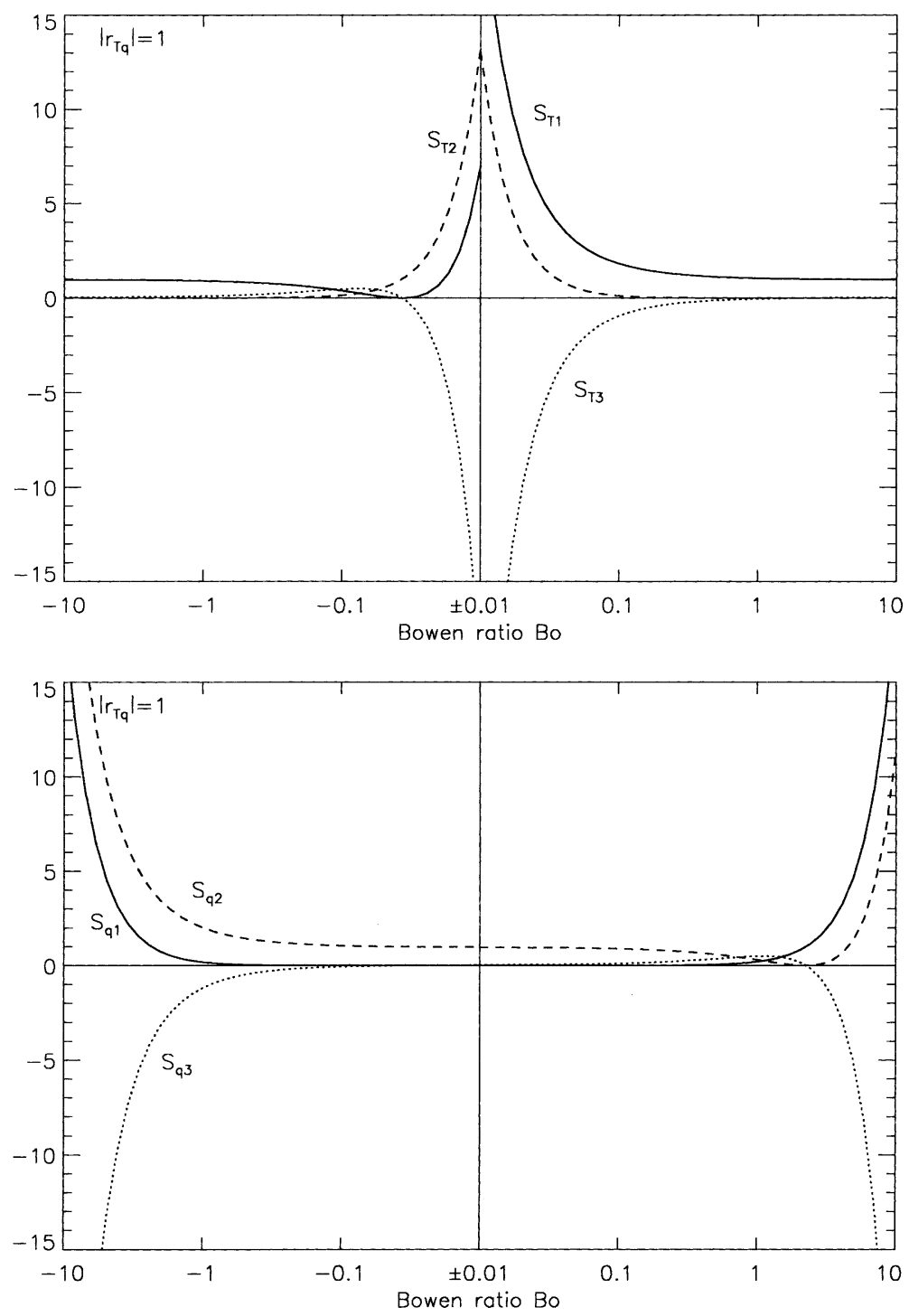

Figure 4. The sensitivity coefficients $S_{T}$ (upper panel) and $S_{q}$ (lower panel) for the two wavelengths $\lambda_{1}=940 \mathrm{~nm}, \lambda_{2}=3.2 \mathrm{~mm}$ and for $\left|r_{T q}\right|=1$. Environment conditions are $T=20{ }^{\circ} \mathrm{C}$, relative humidity of $90 \%$ and $P=1000 \mathrm{hPa}$.

\section{Measurement Results and Discussion}

In order to determine $C_{T q}$, we find from the results of the previous section that uncertainties in $C_{n_{1} n_{2}}$ measurements are rather uncritical, but errors in $C_{n_{1}}^{2}$ and $C_{n_{2}}^{2}$ values should be very small. Due to the fact that these structure parameters are averaged over a long path length of several kilometres, 

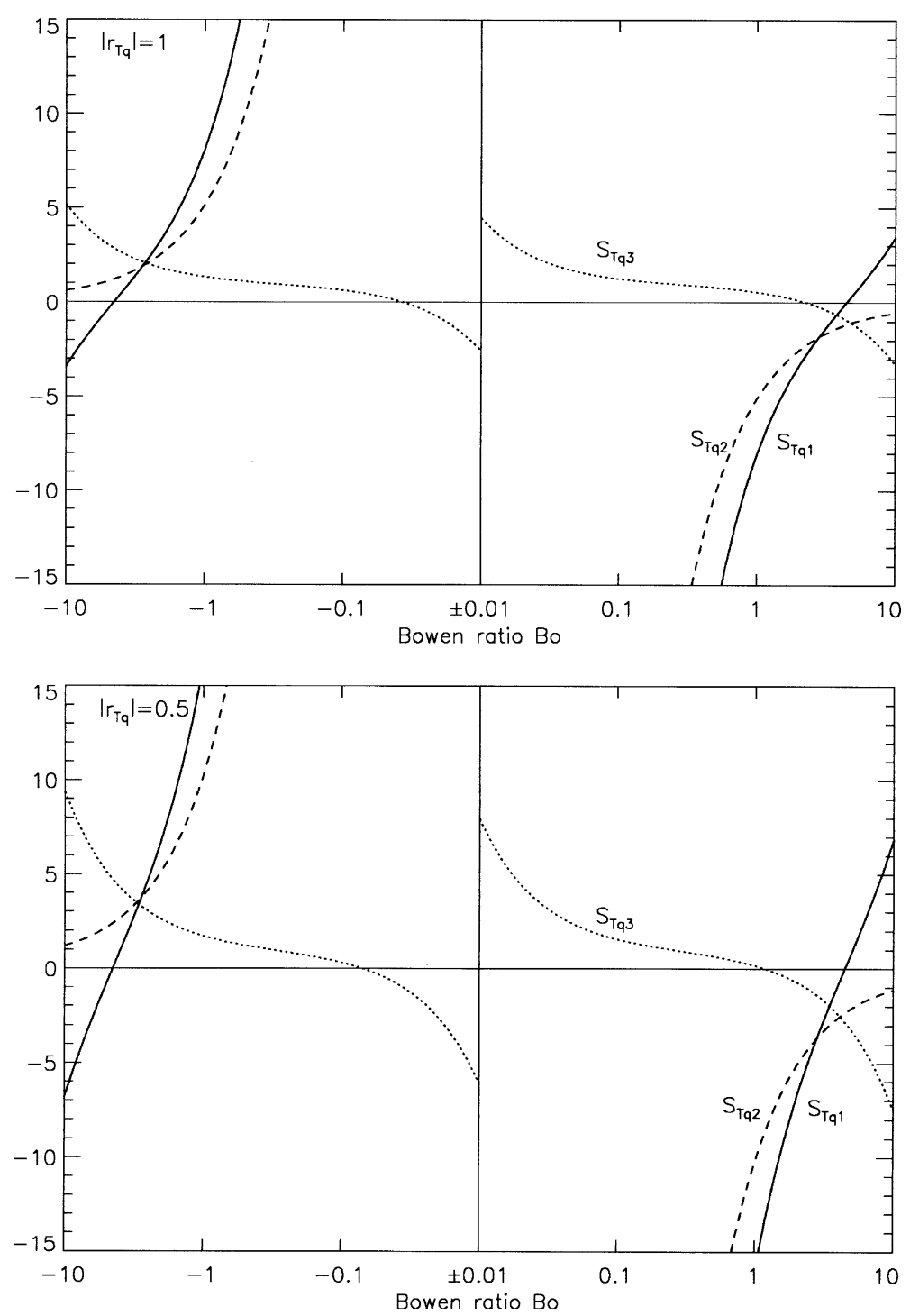

Figure 5. The sensitivity coefficients $S_{T q}$ for $\left|r_{T q}\right|=1$ (upper panel) and for $\left|r_{T q}\right|=0.5$ (lower panel). All other parameters are the same as in Figure 4.

already with rather short averaging times of $10 \mathrm{~min}$ the statistical errors of $C_{n_{1}}^{2}$ and $C_{n_{2}}^{2}$ are small. Similarly as specified by Scintec for the commercial BLS900 instrument, we found experimentally that our optical scintillometer reaches the noise limit at $C_{n}^{2} \approx 5 \times 10^{-17} \mathrm{~m}^{-2 / 3}$. With typical daytime values of $C_{n}^{2} \approx 5 \times 10^{-15} \mathrm{~m}^{-2 / 3}$ (cf. Figure 6) this corresponds to a measurement uncertainty of approximately $1 \%$. Even smaller are the statistical measurement errors in $C_{n_{2}}^{2}$; the noise limit of the microwave scintillometer was found 

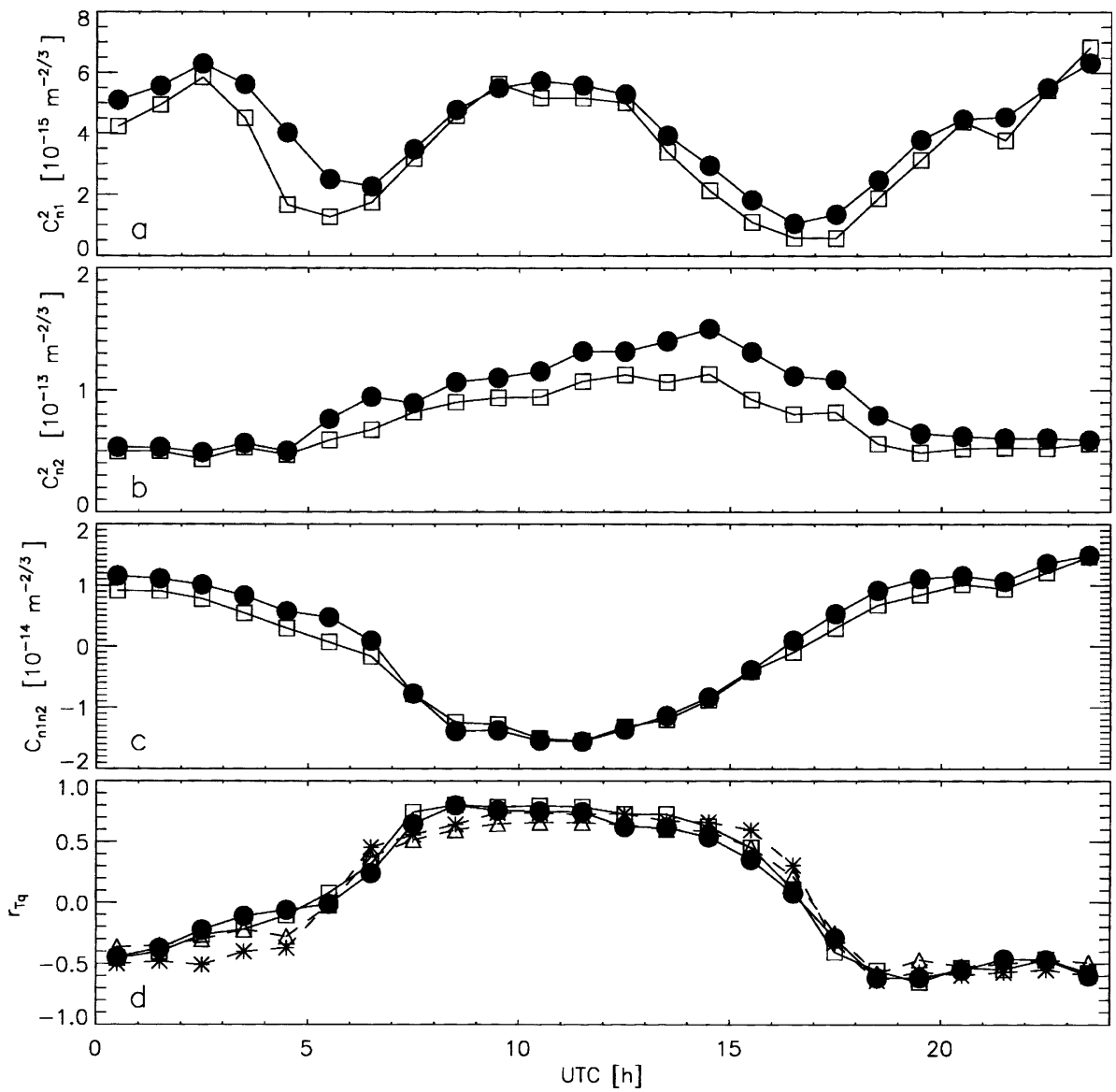

Figure 6. Median (squares) and mean (circles) diurnal cycle of $C_{n_{1}}^{2}$ (panel $a$ ), $C_{n_{2}}^{2}$ (panel $b$ ), $C_{n_{1} n_{2}}$ (panel $c$ ) and the correlation coefficient $r_{T q}$ (panal $d$ ). The dashed cures are median (asterisk) and mean (triangle) values derived by eddy-covariance techniques. The local midday is at 1100 UTC.

to be considerably lower than $10^{-15} \mathrm{~m}^{-2 / 3}$. As typical measurement values are around $10^{-13} \mathrm{~m}^{-2 / 3}$ (cf. Figure 6) the errors are smaller than $1 \%$. The uncertainty of $C_{n_{1} n_{2}}$, derived from the cross-covariance, is larger. It also can be estimated experimentally: the cross-covariance between the two signals should disappear when they are correlated with a time lag. As measurements are not perfect the time-lagged cross-covariance does not absolutely disappear but reaches the noise limit, corresponding to $C_{n_{1} n_{2}} \approx 3 \times 10^{-15} \mathrm{~m}^{-2 / 3}$. With typical $C_{n_{1} n_{2}}$ values (cf. Figure 6) around midnight and midday $( \pm 1.5 \times$ $10^{-14} \mathrm{~m}^{-2 / 3}$ ) this corresponds approximately to statistical errors of $20 \%$. Let us note that this value is in good agreement with the theoretical expectation of the signal-to-noise ratio of the bichromatic correlation (bichromatic 
covariance $B_{P}$ divided by the square root of the two monochromatic variances): the bichromatic correlation was computed numerically and was found to be $0.04 r_{n_{1} n_{2}}$, where $r_{n_{1} n_{2}}=C_{n_{1} n_{2}} /\left(C_{n_{1}}^{2} C_{n_{2}}^{2}\right)^{-1 / 2}$ with values between -1 and +1 . With the above mentioned signal-to-noise ratio of $C_{n_{1}}^{2}$ and $C_{n_{2}}^{2}$ being at least 100 and 200, respectively the detection limit of the bichromatic correlation is then $(1 / 2)(1 / 100+1 / 200) \approx 0.007$. Thus, this limit is at least five times smaller than the bichromatic correlation (since $\left|r_{n_{1} n_{2}}\right|$ is not vanishing), which corresponds well to the empirically found error of $C_{n_{1} n_{2}}$ of $20 \%$.

For the quantification of the temperature-humidity correlation the parameter $r_{T q}$, defined in (2), is best suited. This parameter and the measured refractivity structure parameters are shown in Figure 6 as mean (circles) and median (squares) diurnal cycles for the whole measuring campaign (15 April 2003 to 29 May 2003). Panel $a$ shows $C_{n_{1}}^{2}$ from the optical scintillometer; as expected (e.g. Wesely and Alcaraz, 1973), the optical scintillation is high around midday (1100 UTC) and disappears almost around sunrise (0600 UTC) and sunset (1700 UTC). During the night $C_{n_{1}}^{2}$ is again large. The refractivity structure parameter for microwaves has a less distinct diurnal cycle (Figure 6, panel $b$ ). In general $C_{n_{2}}^{2}$ is larger during daytime. Compared to the optical structure parameter, $C_{n_{2}}^{2}$ is larger by more than one order of magnitude, caused by atmospheric humidity fluctuations. For both structure parameters the mean values (circles) are slightly larger than the median values (squares) because these structure parameters obey a log-normal rather than a normal distribution (e.g. Lüdi and Magun, 2005 and references therein).

One of our key parameters, the cross-structure parameter $C_{n_{1} n_{2}}$, derived from cross-correlating the optical and microwave signals, is shown in panel c. Obviously $C_{n_{1} n_{2}}$ again has a characteristic diurnal cycle, being negative during daytime and positive during nighttime.

From these refractive parameters and mean meteorological quantities (measured at $40 \mathrm{~m}$ height, next to the transmitters), the meteorological structure parameters and $r_{T q}$ (Equation (2)) can be derived (cf. Section 3.3). From panel $d$ (Figure 6) it is seen that the correlation between humidity and temperature fluctuations also has a clear diurnal cycle: the $T-q$ correlation is high and positive during daytime and negative during nighttime. The anticorrelation during nighttime seems to be less pronounced than the positive daytime correlation. From 19 May 2003 to 18 June 2003 in situ fluctuation measurements were performed at the $50 \mathrm{~m}$ level of the meteorological tower using a sonic anemometer-thermometer and an infrared hygrometer. Therefrom, $r_{T q}$ has also been determined by applying the eddy-covariance method, and is shown as dashed curves in Figure 6d. The triangles and the asterisks indicate the mean and median values, respectively. Obviously, the in situ measured correlation coefficients are very similar to those derived by scintillometry, and the small differences are probably due to the different 
measurement periods. Of particular interest is also the fact that again the nighttime anti-correlation is less pronounced than the daytime positive correlation. Similar diurnal cycles of $r_{T q}$ were also observed by other in situ investigations using eddy-covariance instruments (e.g. Andreas et al., 1998).

The determined values of $r_{T q}$ of all usable 10-min samples are shown in Figure 7 as a function of $B o$. As $r_{T q}$ is not directly measured, but computed from $C_{T q}, C_{q}^{2}$ and $C_{T}^{2}$ that are contaminated with errors, some unphysical values of $\left|r_{T q}\right|$ larger than 1 are seen in Figure 7. The Bowen ratio can be determined by measuring temperature and humidity at two heights, and approximating this "gradient-Bo" by (Andreas et al., 1998)

$$
B o=\frac{c_{p}\left(T_{2}-T_{1}\right)}{L_{v}\left(q_{2}-q_{1}\right)} .
$$

For determining the temperature and humidity gradients necessary for the application of (22) we used data from psychrometer measurements at 2, 10, and $40 \mathrm{~m}$ above the ground, We derived $B o$ from the measurements at 2 and $40 \mathrm{~m}$. For $|B o|>0.2$, we used only those values that were equal within a factor of 2 with $B o$ derived from the other two height combinations.

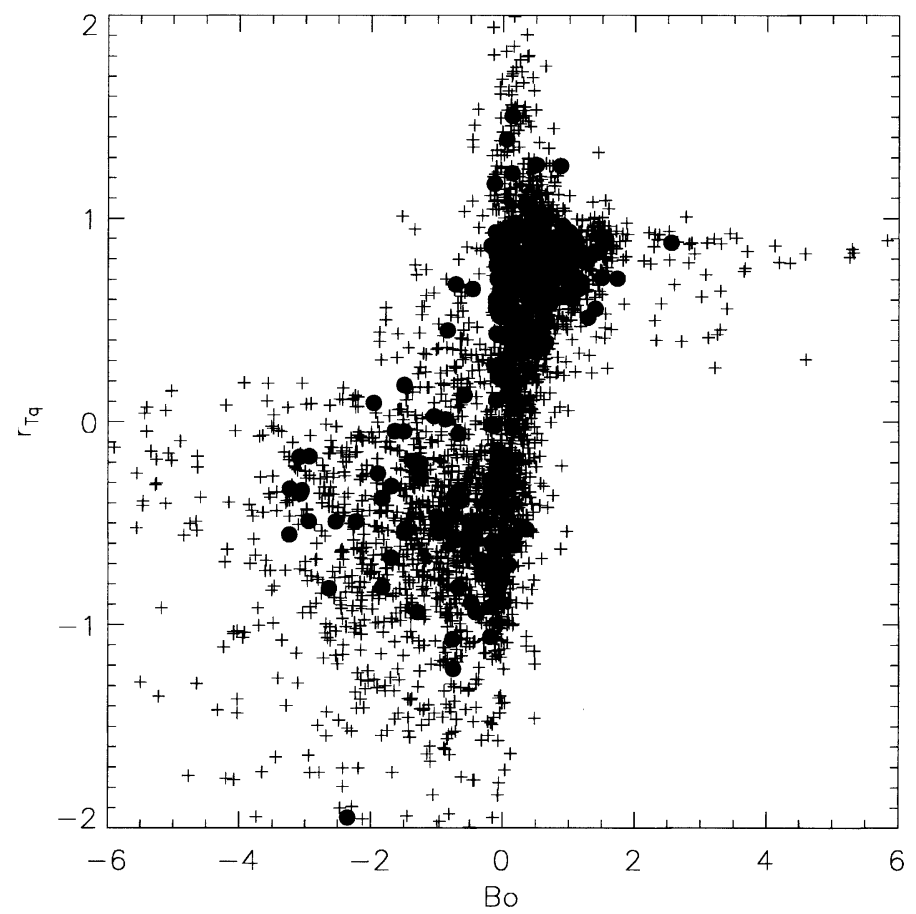

Figure 7. Determined correlation coefficients $r_{T q}$ versus the Bowen ratio Bo. For most points (crosses) Bo was determined by Equation (22). During the period from 19 to 29 May 2003, Bo was also derived from eddy-covariance measurements of the sensible and latent heat fluxes at $50 \mathrm{~m}$ height (circles). 
As mentioned above, starting on May 19, 2003, direct wind, temperature and humidity fluctuation measurements were performed at the $50-\mathrm{m}$ level of the meteorological tower. From these measurements, turbulent fluxes of sensible and latent heat have been determined using the eddy-covariance method, and the $B o$ ratio was directly calculated from the flux data (bold dots in Figure 7). A comparison between the latter Bo and the "gradient-Bo" (Equation (22)) showed that the gradient- $B o$ is reasonably accurate. As expected from the foregoing sensitivity analysis in Section 4 , the scatter of $r_{T q}$ for individual measurements is large, especially for $|B o| \lesssim 1$. Some scatter is likely also due to the fact that the point measure of $B o$ is not representative for the whole propagation path, Nevertheless, a clear relation between $r_{T q}$ and $B o$ can be well observed: the correlation coefficient $r_{T q}$ is close to zero for small $|B o|$ and rises toward 1 for larger $B o$. For negative $B o$ the coefficient $r_{T q}$ becomes also negative. This finding is substantiated in Figure 8 where we divided all measurement values into classes of $B o$ ranges and evaluated the data in each class statistically. For $|B o|<1$ the class were chosen in steps of 0.2 ; for $|B o| \geq 1$ the classes were chosen in steps of 1.0. The mean values (circles in Figure 8) of $r_{T q}$ for each class rise steadily from -0.6 for $B o<-2$ to zero at small $|B o|$ and to $\mathrm{r}_{T q}=0.8$ for $B o>2$. The vertical bars indicate the statistical errors of the mean values (standard deviation divided by the square root of the number of measurements), which are in the order of 0.05 . (cf. Table I). The median values

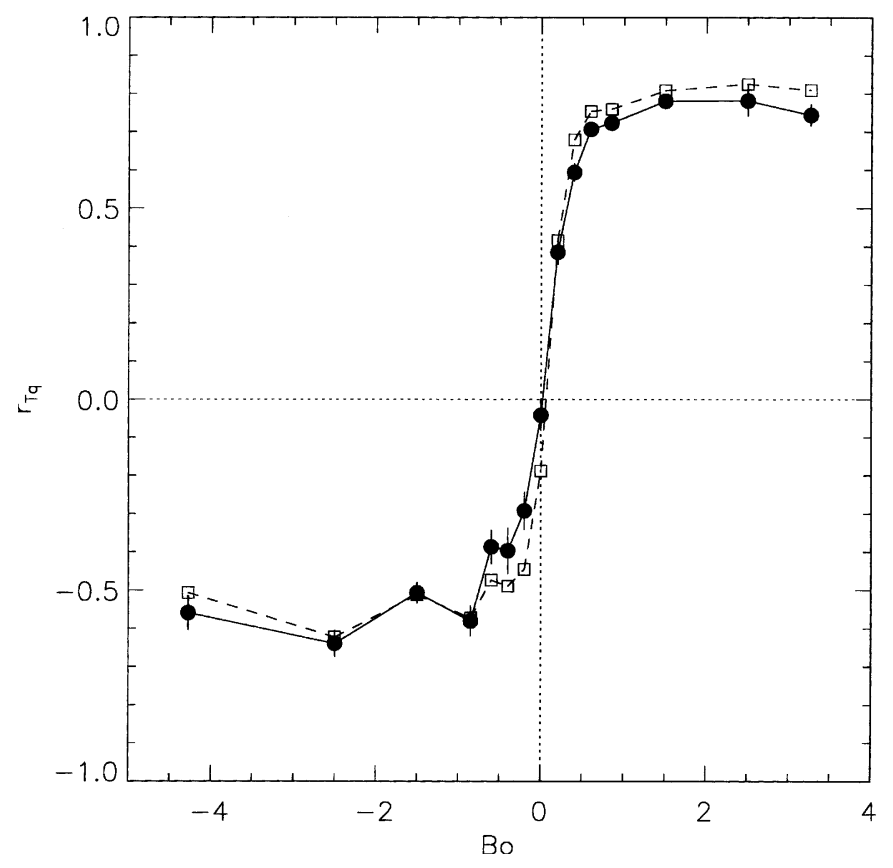

Figure 8. Mean values (circles), their statistical errors (vertical bars) and median values (squares) of $r_{T q}$ for $B o$ classes. The numerical values are summarised in Table I. 
TABLE I

Summary of the statistically averaged $r_{T q}$ values in $B o$ classes. $\overline{r_{T q}}$ and $\left\langle r_{T q}\right\rangle$ are the mean and median values of $r_{T q}$, respectively, $S$ is the standard deviation, $S_{\bar{r}}(=S / \sqrt{n})$ is the mean error of the mean value and $n$ is the number of measurements.

\begin{tabular}{llllll}
\hline Bo & \multicolumn{1}{r}{$\overline{r_{T q}}$} & $\left\langle r_{T q}\right\rangle$ & $S$ & $S_{\bar{r}}$ & $n$ \\
\hline$<-3$ & -0.559 & -0.507 & 0.495 & 0.045 & 123 \\
$-3.0-2.0$ & -0.639 & -0.623 & 0.479 & 0.035 & 191 \\
$-2.0-1.0$ & -0.507 & -0.513 & 0.506 & 0.027 & 340 \\
$-1.0-0.7$ & -0.581 & -0.573 & 0.519 & 0.039 & 176 \\
$-0.7-0.5$ & -0.387 & -0.474 & 0.525 & 0.044 & 141 \\
$-0.5-0.3$ & -0.397 & -0.489 & 0.649 & 0.059 & 118 \\
$-0.3-0.1$ & -0.292 & -0.446 & 0.678 & 0.049 & 188 \\
$-0.1-0.1$ & -0.041 & -0.188 & 0.756 & 0.040 & 357 \\
$0.1-0.3$ & 0.385 & 0.416 & 0.600 & 0.032 & 356 \\
$0.3-0.5$ & 0.593 & 0.679 & 0.481 & 0.023 & 435 \\
$0.5-0.7$ & 0.707 & 0.754 & 0.302 & 0.017 & 288 \\
$0.7-1.0$ & 0.723 & 0.759 & 0.275 & 0.019 & 199 \\
$1.0-2.0$ & 0.781 & 0.808 & 0.164 & 0.012 & 188 \\
$2.0-3.0$ & 0.781 & 0.825 & 0.179 & 0.040 & 20 \\
$\geq 3$ & 0.744 & 0.809 & 0.191 & 0.029 & 45 \\
\hline
\end{tabular}

(squares) are also shown in Figure 8. The latter ones and the mean values are basically equal to within the statistical errors, indicating that $r_{T q}$ seems to be normally distributed, This was also confirmed by the fact that the higher moments (particularly the skewness) were close to zero for each sample population of the $B o$-Classes. Of particular interest is the fact that the three $r_{T q}$ values at $B o>1$ are equal within the error bars, namely $r_{T q}=0.78 \pm 0.01$, $r_{T q}=0.78 \pm 0.04$, and $r_{T q}=0.74 \pm 0.03$, indicating that $r_{T q}$ reaches an upper limit. Also for negative $B o$ the anti-correlation reaches a minimal value: the two $r_{T q}$ values at $B o<-2$ are also equal within the error bars, i.e. $r_{T q}=-0.64 \pm 0.04$ and $r_{T q}=-0.56 \pm 0.05$. Obviously, as already mentioned above, the negative correlation is less pronounced. All measurement points in Figure 8, including the statistical errors, are summarised in Table I.

Even though there are quite large uncertainties in the behaviour and absolute values of $r_{T q}$, there do not exist many investigations into the correlation of temperature-humidity fluctuations. In general, it is believed that when sensible and latent heat fluxes have the same direction (i.e. $B o>0$ ), the correlation between humidity and temperature fluctuations is positive. On the other hand, when sensible and latent heat fluxes have an opposite flowdirection (i.e. $B o<0$ ), humidity and temperature fluctuations are anti-correlated. In a flow that strictly obeys MOST, the correlation coefficient 
between any two conservative scalars should be \pm 1 (Hill, 1989). However, this is not always true, and MOST can be violated (Hill, 1989; Andreas et al., 1998). It is thought that scalar-scalar correlations, such as $r_{T q}$, are especially sensitive indicators of deviations from MOST (Hill, 1989). Over the metrescale heterogeneous landscape "Sevilleta", Andreas et al. (1998) measured with an in situ eddy-covariance instrument values of $r_{T q}$ being 0.8 or less with a mean value of 0.76 for daytime measurements. Consequently it was concluded that violations of MOST are due to the metre-scale heterogeneity of the Sevilleta. The heterogeneity at our test site is higher than at the Sevilleta, as the dominant heterogeneity scales are in the order of hundreds of metres. Nevertheless our measured values of $r_{T q}$ are similar. The mean value of $r_{T q}$ of 0.8 for large $B o$ is slightly larger than their 0.76 .

From other investigations, there exist several hypotheses to explain the non-perfect temperature-humidity correlation observed elsewhere (cf. Priestley and Hill, 1985; De Bruin et al., 1993; Andreas et al., 1998). In our case, the explanation of Andreas et al. (1998) for the Sevilleta landscape appears to be most plausible, as the heterogeneity leads to distributed heat and moisture sources that cannot produce temperature and humidity fluctuations with perfect correlation.

Finally, as mentioned in the introduction, let us close this section by noting that measured $r_{T q}$ (or $C_{T q}$ ) lead to better estimates of $C_{T}^{2}$ and $C_{q}^{2}$, yielding more accurate heat flux estimates. For daytime non-stable stratification $\left(r_{T q}>0, B o>0\right), C_{T}^{2}$ and $C_{q}^{2}$, was determined by the conventional two wavelength method (assuming $r_{T q}=1$ ) and was compared to the respective structure parameters determined by our method, The more $r_{T q}$ deviates from 1 the worse is the estimation by the two-wavelength method. Assuming $r_{T q}=1$ instead of $r_{T q}=0.8$ leads to an overestimation of $C_{q}^{2}$ by a factor of around 1.16 and an underestimation of $C_{T}^{2}$, by a factor of 1.03 . For values of $\mathrm{r}_{T q}$ between 0.4 and 0.6 the conventional method leads to a $C_{q}^{2}$ overestimation and $C_{T}^{2}$ underestimation by factors of 1.25 and 1.1, respectively. For $r_{T q}$ smaller than 0.4 the overestimation of $C_{q}^{2}$ remains approximately around a factor of 1.25 but the underestimation of $C_{T}^{2}$ is raised to a factor of 1.2.

The fact that $C_{q}^{2}$ is notably overestimated by the two-wavelength method, even when the deviation from perfect positive $r_{T q}$ correlation is only small $\left(r_{T q} \approx 0.8\right)$, can perhaps partly explain the conclusions of Beyrich et al. (2004) - that is, that conventionally derived scintillometric latent heat fluxes in Lindenberg have been found to be significantly larger than respective in situ measurements.

\section{Conclusion}

We investigated theoretically and experimentally the measurability of $C_{T q}$ and $r_{T q}$ by cross-correlating the detected signals of an optical and a 
microwave scintillometer. For the first time (as far as we know), it was possible to measure path-averaged correlations of temperature-humidity fluctuations. Compared to the traditional two-wavelength method the necessary instrumental modifications are marginal. Hence, in comparison to the suggested three (or more) wavelength method by Andreas (1990), which also measures path-averaged $C_{T q}$ values, our method is less expensive and simpler in set-up. However, unlike the preliminary hopes mentioned in Lüdi (2002), the sensitivity analysis showed that our method does no better in measuring $C_{T q}$ than the three-wavelength method. Therefore, the errors in individual measurements can be large. The correlation of temperature-humidity fluctuations derived by scintillometry was compared to that derived by eddy-covariance, and good agreement was found. Our results are similar to those found over the (metre-scale) heterogeneous Sevilleta by Andreas et al. (1998), as $r_{T q}$ fell to -0.6 for $B o$ smaller than -2 and rose to a value of 0.8 for $B o>1$. The statistical errors in the averaged $r_{T q}$ in each $B o$ class are approximately 0.05 , which is quite small.

The fact that $r_{T q}$ is smaller than 1 shows that our observations violate MOST. However, the quite large temperature-humidity (anti-)correlation for $|B o|>1$ also indicates that MOST is not seriously violated, as also concluded for the Sevilleta data (Andreas et al., 1998). Therefore, in order to infer heat fluxes from scintillometer observations made over heterogeneous landscapes, the similarity functions of MOST might still be applicable. However, when the common two-wavelength method is used, we suggest that non-perfect correlation of temperature-humidity fluctuations should be presumed. For Lindenberg and for similar landscapes (as encountered in large parts of northern central Europe south of the Baltic Sea) the function found in Figure 8 (and Table I) may serve as a reference function.

\section{Appendix A. Sensitivity Functions}

The explicit form of the sensitivity functions shown in Figures 4 and 5 are given below:

$$
\begin{aligned}
& S_{T 1}=\frac{\left(A_{1}^{2}+2 \frac{A_{1} B_{1}\left|r_{T}\right|}{K B o}+\frac{B_{1}^{2}}{K^{2} B o^{2}}\right) B_{2}^{2}}{\left(A_{1} B_{2}-A_{2} B_{1}\right)^{2}}, \\
& S_{T 2}=\frac{\left(A_{2}^{2}+2 \frac{A_{2} B_{2}\left|r_{T q}\right|}{K B o}+\frac{B_{2}^{2}}{K^{2} B 0^{2}}\right) B_{1}^{2}}{\left(A_{1} B_{2}-A_{2} B_{1}\right)^{2}},
\end{aligned}
$$




$$
\begin{aligned}
& S_{T 3}=-2 \frac{\left(A_{1} A_{2}+\frac{\left(A_{1} B_{2}+A_{2} B_{1}\right)\left|r_{T q}\right|}{K B o}+\frac{B_{1} B_{2}}{K^{2} B o^{2}}\right) B_{1} B_{2}}{\left(A_{1} B_{2}-A_{2} B_{1}\right)^{2}}, \\
& S_{T q 1}=-\frac{\left(\frac{A_{1}^{2} B o K}{\left|r_{T q}\right|}+2 A_{1} B_{1}+\frac{B_{1}}{\left|r_{T q}\right| B o K}\right) A_{2} B_{2}}{\left(A_{1} B_{2}-A_{2} B_{1}\right)^{2}}, \\
& S_{T q 2}=-\frac{\left(\frac{A_{2}^{2} B o K}{\left|r_{T q}\right|}+2 A_{2} B_{2}+\frac{B_{2}}{\left|r_{T q}\right| B o K}\right) A_{1} B_{1}}{\left(A_{1} B_{2}-A_{2} B_{1}\right)^{2}}, \\
& S_{T q 3}=\frac{\left(\frac{A_{1} A_{2} B o K}{\left|r_{T q}\right|}+A_{1} B_{2}+A_{2} B_{1}+\frac{B_{1} B_{2}}{\left|r_{q q}\right| B o K}\right)\left(A_{1} B_{2}+A_{2} B_{1}\right)}{\left(A_{1} B_{2}-A_{2} B_{1}\right)^{2}}, \\
& S_{q 1}=\frac{\left(A_{1}^{2} K^{2} B o^{2}+2 A_{1} B_{1}\left|r_{T q}\right| K B o+B_{1}^{2}\right) A_{2}^{2}}{\left(A_{1} B_{2}-A_{2} B_{1}\right)^{2}}, \\
& S_{q 2}=\frac{\left(A_{2}^{2} K^{2} B o^{2}+2 A_{2} B_{2}\left|r_{T q}\right| K B o+B_{2}^{2}\right) A_{1}^{2}}{\left(A_{1} B_{2}-A_{2} B_{1}\right)^{2}}, \\
& S_{q 3}=-2 \frac{\left(A_{1} A_{2} K^{2} B o^{2}+\left(A_{1} B_{2}+A_{2} B_{1}\right)\left|r_{T q}\right| K B o+B_{1} B_{2}\right) A_{1} A_{2}}{\left(A_{1} B_{2}-A_{2} B_{1}\right)^{2}} .
\end{aligned}
$$

\section{B. Absorption-induced Fluctuations}

Sensitive to possible absorption-induced fluctuations are the mm-wave variance, yielding $C_{n_{2}}^{2}$, and the cross covariance, yielding $C_{n_{1} n_{2}}$. In order to estimate the influence of absorption on scintillation at $94 \mathrm{GHz}$, the analysis of Nieveen et al. (1998) and Hill et al. (1980) are useful. The variance of the detected signal can be regarded as the sum of three variances, namely $\sigma_{R}^{2}$ due to the real part $n$ of the refractive-index fluctuations; $\sigma_{I}^{2}$, due to the imaginary part $m$ of the refractive-index fluctuations; and $\sigma_{I R}$, due to the covariance of the real and imaginary parts of the refractive-index fluctuations. (In Nieveen et al. (1998) the latter was not considered.) They are given by

$$
\begin{aligned}
& \sigma_{R}^{2}=8.448 \pi^{2} k_{1} k_{2} \int_{0}^{L} d x \int_{0}^{\infty} d \kappa \kappa^{-8 / 3} \cdot F \cdot A \\
& \sigma_{I}^{2}=8.448 \pi^{2} k_{1} k_{2} \int_{0}^{L} d x \int_{0}^{\infty} d \kappa \kappa^{-8 / 3} \cdot F \cdot B
\end{aligned}
$$




$$
\sigma_{I R}=8.448 \pi^{2} k_{1} k_{2} \int_{0}^{L} d x \int_{0}^{\infty} d \kappa \kappa^{-8 / 3} \cdot F \cdot C,
$$

with

$$
\begin{aligned}
& A=\left(\sin \left(\frac{\gamma(L-x) \kappa^{2}}{2 k_{1}}\right)\right)^{2} C_{n}^{2}, \\
& B=\left(\cos \left(\frac{\gamma(L-x) \kappa^{2}}{2 k_{1}}\right)\right)^{2} C_{m}^{2}, \\
& C=2\left(\cos \left(\frac{\gamma(L-x) \kappa^{2}}{2 k_{1}}\right)\right)\left(\sin \left(\frac{\gamma(L-x) \kappa^{2}}{2 k_{1}}\right)\right) C_{n m},
\end{aligned}
$$

where $k_{1}=k_{2}$ is the wavenumber of the $\mathrm{mm}$ wave. All parameters are defined as in Section 3. Neglecting temperature fluctuations, the computation (with MPM93 and the in situ meterological data) of the ratios $C_{m}^{2} / C_{n}^{2}$ and $C_{n m} / C_{n}^{2}$ are found to be smaller than $4 \times 10^{-7}$ and $6 \times 10^{-4}$, respectively. The boundaries of the integration over the spatial wave number $\kappa$, in (B.1) to (B.3) can be narrowed by temporal filtering of the measured signal with an appropriate band-pass filter (cf. Section 2). In our experiment the integration boundaries are approximately at $\kappa \cong 0.04$ and $20 \mathrm{~m}^{-1}$, respectively, and with these integration limits it is found that $\sigma_{I}^{2} / \sigma_{R}^{2} \lesssim 0.0003$ and $\sigma_{I R} / \sigma_{R}^{2} \lesssim 0.001$. Thus, absorption effects at the mmwave variance can be safely neglected. The same was found by Wouter Meijninger (personal communication, 2004) who also computed the absorption effects at $94 \mathrm{GHz}$, following Nieveen et al. (1998). The small influence of absorption at $94 \mathrm{GHz}$ is due to the fact that this frequency is in an atmospheric absorption window.

\section{Acknowledgements}

The authors thank Lorenz Martin (IAP, University of Bern) and Ulrich Weisensee (MOL, DWD) for contributing to the set-up of the measurements and maintaining the technical equipment, as well as Jens-Peter Leps (MOL, DWD) for processing the in situ tower data. We acknowledge Armasuisse (VBS) for partial funding and support of this project under contract number 4500310096 . We finally thank three anonymous reviewers of the manuscript for their critical remarks on an earlier version that helped to improve the presentation. 


\section{References}

Andreas, E. L.: 1987, 'On the Kolmogorov constants for the temperature-humidity Cospectrum and the Refractive Index Spectrum', J. Atmos. Sci. 44, 2399-2406.

Andreas, E. L.: 1988, 'Estimating $C_{n}^{2}$ over Snow and Sea Ice from Meteorological Data', J. Opt. Soc. Amer. A 5, 481-495.

Andreas, E. L.: 1989, 'Two-wavelength Method of Measuring Path-averaged Turbulent Surface Heat Fluxes', J. Atmos. Ocean. Tech. 6, 280-292.

Andreas, E. L.: 1990, 'Three-wavelength Method of Measuring Path-averaged Turbulent Heat Fluxes', J. Atmos. Ocean. Tech. 7, 801-814.

Andreas, E. L., Hill, R. J., Gosz, J. R., Moore, D. I., Otto, D. W. and Sarma, A. D.: 1998, 'Statistics of Surface-layer Turbulence over Terrain with Metre-scale Heterogeneity', Boundary-Layer Meteorol. 86, 379-408.

Beyrich, F., Bange, J., Bernhofer, C., Foken, T., Hennemuth, B., Huneke, S., Kohsiek, W., Leps, J.-P., Lohse, H., Lüdi, A., Mauder, M. and Meijninger, W. M. L.: 2004, 'Energy and Water Vapour Fluxes over a Heterogeneous Land Surface: The LITFASS-2003 Experiment', in Proceedings of the 16th Symposium on Boundary Layers and Turbulence, Portland, Maine U.S.A.

Beyrich, F., De Bruin, H. A. R., Meijninger, W. M. L., Schipper, W. and Lohse, H.: 2002, 'Results from One-year Continuous Operation of a Large Aperture Scintillometer over a Heterogeneous Land Surface', Boundary-Layer Meteorol. 105, 85-97.

Caccia, J. L., Azouit, M. and Vernin, J.: 1987, 'Wind and $C_{n}^{2}$ Profiling by Single-star Scintillation Analysis', Apple Opt. 26, 1288-1294.

De Bruin, H. A. R., Kohsiek, W. and Den Hurk, B. J. J. M.: 1993, 'A Verification of Some Methods to Determine the Fluxes of Momentum, Sensible Heat and Water Vapour using Standard Deviation and Structure Parameter of Scalar Meteorological Quantities', Boundary-Layer Meteorol. 63, 231-257.

Green, A. E., Astill, M. S., McAneney, K. J. and Nieveen, J. P.: 2001, 'Path-averaged Surface Fluxes Determined from Infrared and Microwave Scintillometers', Agric. For. Meteorol. 109, 233-247.

Green, A. E., Green, S. R., Astill, M. S. and Caspari, H. W.: 2000, 'Estimating Latent Heat Flux from a Vineyard Using Scintillometry', J. Terr. Atmos. Ocean. Sci. 11(2), 525-542.

Hill, R.J.: 1988, 'Comparison of Scintillation Methods for Measuring the Inner Scale of Turbulence', Appl. Opt. 27, 2187-2193.

Hill, R. J.: 1989, 'Implications of Monin-Obukhov Similarity Theory for Scalar Quantities', J. Atmos. Sci. 46, 2236-2244.

Hill, R. J.: 1997, 'Algorithms for Obtaining Atmospheric Surface-layer Fluxes from Scintillation Measurements', J. Atmos. Ocean. Tech. 14, 456-467.

Hill, R. J., Bohlander, R. A., Clifford, S. F., McMillan, R. W., Priestley, J. T. and Schoenfeld, W. P.: 1988, 'Turbulence-induced Millimeter-wave Scintillation Compared with Micrometeorological Measurements', IEEE Trans. Geosci. Remote Sens. 26, 330-342.

Hill, R. J., Clifford, S. F. and Lawrence, R. S.: 1980, 'Refractive-index and Absorption Fluctuations in the Infrared Caused by Temperature, Humidity, and Pressure Fluctuations', J. Opt. Soc. Amer. 70, 1192-1205.

Hill, R. J. and Lataitis, R. J.: 1989, 'Effect of Refractive Dispersion on the Bichromatic Correlation of Irradiances for Atmospheric Scintillation', Appl. Opt. 28, 4121-4125.

Ishimaru, A.: 1978, 'Wave Propagation and Scattering in Random Media,' Vol. 2, Academic Press, New York, San Francisco, 339 pp.

Kohsiek, W. and Herben, A. J.: 1983, 'Evaporation Derived from Optical and Radio-wave Scintillation', Appl. Opt. 22, 2566-2569. 
Liebe, H. J., Hufford, G. A., and Cotton, M. G.: 1993,'Propagation Modeling of Moist Air and Suspended Water/Ice Particles at Frequencies Below $1000 \mathrm{GHz}$, in: AGARD 52nd Specialists' Meeting of the Electromagnetic Wave Propagation Panel. Mallorca, Spain.

Lüdi, A.: 2002, 'Determination of the Structure Constants of Temperature, Humidity and temperature-humidity by Scintillometry at two Wavelengths', IAP Research Report 200213, Institute of Applied Physics, University of Bern, Bern, Switzerland. (in German), 8 pp.

Lüdi, A. and Magun, A.: 2005, 'Refractivity Structure Constant and Length Scales from mm Wave Propagation in the Stably Stratified Troposphere', J. Atmos. Sol. Terr. Phys. 67, 435-447.

Martin, L., Mätzler, C., and Kämpfer, N.: 2000, 'Transmission Measurements at $94 \mathrm{GHz}$ to Validate the Liebe Model', in AP 2000 Millennium Conference on Antennas and Propagation, SP-444, ESA Publication Division. Davos, Switzerland.

Meijninger, W. M. L., Green, A. E., Hartogensis, O. K., Kohsiek, W., Hoedjes, J. C. B., Zuurbier, R. M. and De Bruin, H. A. R.: 2002a, 'Determination of Area-averaged Water Vapour Fluxes with Large Aperture and Radio Wave Scintillometers over a Heterogeneous Surface Flevoland Field Experiment', Boundary-Layer Meteorol. 105, 63-83.

Meijninger, W. M. L., Hartogensis, O. K., Kohsiek, W., Hoedjes, J. C. B., Zuurbier, R. M. and De Bruin, H. A. R.: 2002b, 'Determination of Area-averaged Sensible Heat Fluxes with a Large Aperture Scintillometer over a Heterogeneous Surface - Flevoland Field Experiment', Boundary-Layer Meteorol. 105, 37-62.

Moene, A. F., Meijninger, W. M. L., Hartogensis, O. K., Heusinkveld, B. G., and de Bruin, H. A. R.: 2005, 'The Effects of Finite Accuracy in the Manufacturing of large Aperture Scintillometers', Internal report 2005/1, Wageningen University, Meteorology and Air Quality Group, Wageningen, The Netherlands, 19 pp.

Nieveen, J. P., Green, A. E. and Kohsiek, W.: 1998, 'Using a Large-aperture Scintillometer to Measure Absorption and Refractive Index Fluctuations', Boundary-Layer Meteorol. 87, 101-116.

Ochs, G. R. and Hill, R. J.: 1982, 'A Study of Factors Influencing the Calibration of Optical $C_{n}^{2}$ Meters', NOAA Tech. Memo. ERL WPL-106, NOAA, 24 pp.

Ochs, G. R. and Wang, T. I.: 1978, 'Finite Aperture Optical Scintillometer for Profiling Wind and $C_{n}^{2}$, Appl. Opt. 17, 3774-3778.

Phelps, G. T. and Pond, S.: 1971, 'Spectra of the Temperature and Humidity Fluctuations and the Turbulent Fluxes of Momentum, Moisture and Sensible Heat over the Ocean', J. Atmos. Sci. 28, 918-928.

Priestley, J. T. and Hill, R. J.: 1985, 'Measuring High-frequency Humidity, Temperature and Radio Refractive Index in the Surface Layer', J. Atmos. Ocean. Tech. 60, 233-251.

Tatarskii, V. I.: 1971, 'The Effects of the Turbulent Atmosphere on Wave Propagation,' Isreal Program for Scientific Translation Ltd., Jerusalem, 472 pp.

Wang, T. I., Ochs, G. R. and Clifford, S. F.: 1978, 'A Saturation-resistant Optical Scintillometer to Measure $C_{n}^{2}$, J. Opt. Soc. Amer. 68, 334-338.

Wesely, M. L. and Alcaraz, E. C.: 1973, 'Diurnal Cycles of the Refractive Index Structure Function Coefficient', J. Geophys. Res. 78, 6224-6232. 